\title{
ATLAS toward the High Luminosity era: challenges on electronic systems
}

\author{
Junjie Zhu on behalf of the ATLAS Collaboration \\ Department of Physics \\ University of Michigan \\ Ann Arbor, MI, USA \\ junjie@umich.edu
}

\begin{abstract}
To maximize the physics reach, the Large Hadron Collider (LHC) plans to increase its instantaneous luminosity to $7.5 \times 10^{34} \mathrm{~cm}^{-2} \mathrm{~s}^{-1}$ and deliver $3-4 \mathbf{a b}^{-1}$ of data at a centerof-mass energy of $14 \mathrm{TeV}$. To profit from this high-luminosity LHC operation with a pile-up up to 200 inelastic collisions per bunch crossing, performance of the ATLAS detector needs to be maintained and in many systems, improved. The upgraded detector will have an unprecedented output data rate of up to $200 \mathrm{~TB} / \mathrm{s}$. Real-time processing of this large data volume in a short time period is extremely challenging. New sets of both front-end and back-end electronics are required for all subdetectors. The large number of detector channels, huge volumes of input and output data, short time available to process and transmit data, harsh radiation environment, and the need of low power consumption all impose great challenges on the designs of electronic systems.

Index Terms-ATLAS, HL-LHC, electronics, TDAQ, ASIC, FPGA
\end{abstract}

\section{INTRODUCTION}

The Large Hadron Collider (LHC) [1] at CERN is the world's highest-energy particle collider. It has run successfully over the last ten years with the discovery of the Higgs boson being the highlight. The High Luminosity LHC (HL-LHC) [2] is an upgrade of the LHC to increase the $p p$ collision data sample by an order of magnitude to reach $3-4 \mathrm{ab}^{-1}$. The peak instantaneous luminosity will be increased to $7.5 \times 10^{34}$ $\mathrm{cm}^{-2} \mathrm{~s}^{-1}$, compared to the nominal value of $10^{34} \mathrm{~cm}^{-2} \mathrm{~s}^{-1}$ for the LHC. The large dataset delivered by the HL-LHC will substantially boost the accelerator's potential for new physics discoveries and greatly improve the precision to study the properties of the Higgs boson.

The HL-LHC era offers exciting physics possibilities, albeit with substantial experimental challenges. Performance of the ATLAS (A Toroidal LHC ApparatuS) and CMS (Compact Muon Solenoid) detectors in terms of acceptance, efficiency, resolution, and background rejection for all the physics objects needs to be maintained and in many systems, improved. Both ATLAS and CMS collaborations have programs ongoing to upgrade detector, front-end and back-end electronics, and trigger and data acquisition (TDAQ) system to handle the harsh conditions of the HL-LHC.

This works is supported by the US Department of Energy under contract DE-AC02-98CH10886.

\section{ATLAS DETECTOR UPGRADES FOR THE HL-LHC}

The increases in both the integrated and the instantaneous luminosities have significant impacts on the detectors. Charged particles, neutrons and photons produced in the collisions produce ionization and undergo electromagnetic and nuclear interactions in the detectors they pass through and cause radiation damages. The increase of the integrated luminosity implies an increased radiation dose. The higher instantaneous luminosity means many more collisions occur in the same bunch crossing. At the HL-LHC, the average number of interactions in a single crossing will increase from 25 to 200. As a consequence, the detectors at the HL-LHC will have to handle higher particle densities leading to higher detector channel occupancies and higher radiation levels.

ATLAS [3] will replace its inner tracker with a highergranularity all-silicon tracking system consisting of four pixel layers followed by three short-strip layers and two long-strip layers in the barrel region [4] [5]. The forward region will be covered by six pixel disks and seven strip disks. The new tracker will be approximately $6 \mathrm{~m}$ long, around $2 \mathrm{~m}$ in diameter, and covers up to a pseudo-rapidity of $|\eta|=4$. For the pixel sensor two different pitch sizes are considered, either $50 \times 50 \mu \mathrm{m}^{2}$ or $25 \times 100 \mu \mathrm{m}^{2}$. The strip pitch for the barrel region is approximately $74.5 \mu \mathrm{m}$ while the pitch for the endcap region is in the range of $65-85 \mu \mathrm{m}$. The barrel strip length is either $24.1 \mathrm{~mm}$ or $48.2 \mathrm{~mm}$ and the endcap strip length is $69.9-80.7 \mathrm{~mm}$. The total active silicon area is $12.7 \mathrm{~m}^{2}(165$ $\mathrm{m}^{2}$ ) for the pixel (strip) system. The total number of readout channels is 5 billion (60 million) for the pixel (strip) system.

ATLAS plans to add a new high granularity timing detector (HGTD) based on silicon Low Gain Avalanche Detector (LGAD) technology [6]. At the HL-LHC, an average pileup density of 1.8 vertices $/ \mathrm{mm}$ is expected. The HGTD detector can perform high-precision time measurement of all the tracks associated to the primary vertex to suppress pileup collisions and to improve physics object reconstructions. In addition, it offers unique capabilities to measure both the online luminosity on a bunch-by-bunch basis and the integrated luminosity offline. It will be installed in the space between the inner tracker and the endcap calorimeters, and covers the region $2.4<|\eta|<4.0$ with $R_{\min }=12 \mathrm{~cm}$ and $R_{\max }=64 \mathrm{~cm}$. The target time resolution per track, combining multiple hits, 
is from 30 ps at the start of the lifetime to 50 ps after $4 \mathrm{ab}^{-1}$, due to detector aging.

ATLAS will replace the endcap innermost muon station with a New Small Wheels (NSW) detector [7] using smallstrip Thin Gap Chamber (sTGC) and Micro-Mesh Gaseous Structure (MM) chambers before the HL-LHC starts. For the HL-LHC upgrades [8], a new layer of Resistive Plate Chambers (RPC) will be installed on the barrel inner station to increase the RPC trigger efficiency in the barrel region. The new BI RPCs will have a thinner gas gap than the old RPCs (1 $\mathrm{mm}$ instead of $2 \mathrm{~mm}$ ), To accommodate this additional RPC layer, the present Monitored Drift Tube (MDT) chambers need to be replaced by new small-diameter MDT (sMDT) chambers with the tube diameter reduced by half. This is not needed for the large sectors since there is sufficient space available to add the new RPCs without replacing the MDTs.

\section{ELECTRONICS UPGRADE FOR THE HL-LHC}

At the HL-LHC, the average number of inelastic $p p$ interactions per collision increases by a factor of eight, increasing the probability that some interesting physics processes happened during each collision. To profit from this, trigger rates need to be increased and more events need to be recorded for offline analysis. However, many more particles produced in each collision will cause higher detector occupancies and could confuse the trigger system. It will also increase the average event size by $80 \%$.

The ATLAS TDAQ system at the HL-LHC [9] (shown in Fig. 1) will include a single-level hardware trigger (L0 trigger) and an Event Filter (EF) system. The L0 trigger is based on muon and calorimeter information and has a maximum latency of $10 \mu \mathrm{s}$ so that more complex trigger algorithms than in the present first-level system $(2.5 \mu \mathrm{s})$ are feasible. Following the Level-0 trigger decision (LOA), detector data are transmitted to the DAQ system at a rate of $1 \mathrm{MHz}$. The EF system is composed of a processing farm and a hardwaretrack-trigger subsystem (HTT) and will further reduce the event rate for permanent storage to $10 \mathrm{kHz}$. Compared to the present detector, the first-level trigger rate (maximum latency) will be increased by a factor of 10 (4) and the output event rate for storage will be increased by a factor of 10 . As a result, the upgraded DAQ system needs to have a total bandwidth of 5.2 TB/s, compared to $0.29 \mathrm{~TB} / \mathrm{s}$ for the present DAQ system.

The on-detector front-end electronics will be exposed to much higher radiation levels during the HL-LHC operation. Latest technology advancements will be exploited to use new products and implement robust architectures to operate these electronics for about 15 years. In addition, both calorimeter and muon systems will send off detector data continuously (or triggerless) to back-end electronics so that more information can be passed to the trigger system. Large processing power and high throughput are needed for these front-end electronics.

Both front-end and back-end electronics for almost all subdetector systems need to be replaced to be compatible with the upgraded TDAQ system. Good progress has been made on the developments of electronic prototypes for all sub-detector systems. A short and incomplete summary of electronics 1 upgrades planned for the HL-LHC are listed below:

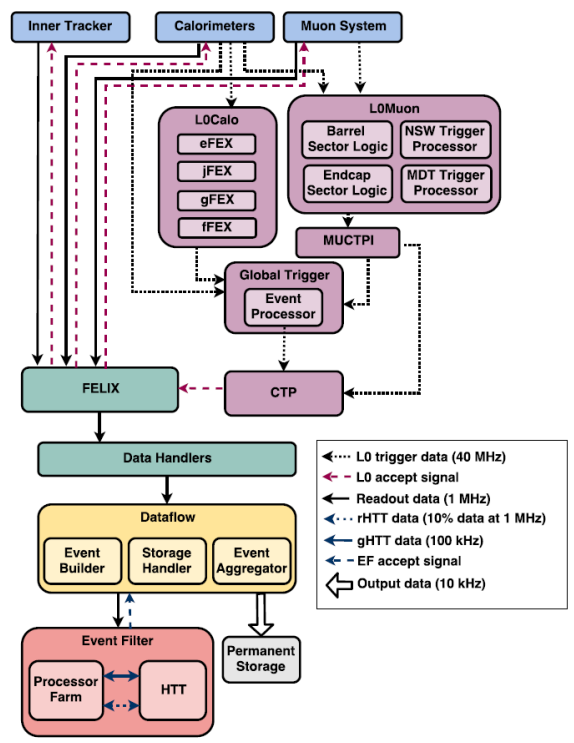

Fig. 1. Overall design of the ATLAS TDAQ system at the HL-LHC [9].

\section{A. Silicon Pixel electronics}

A front-end ASIC [10] based on $65 \mathrm{~nm}$ CMOS technology (shown in Fig. 2) is being developed to provide robust operation for the pixel detector at the HL-LHC, with an estimated hit rate of $3 \mathrm{GHz} / \mathrm{cm}^{2}$ or $75 \mathrm{kHz}$ per pixel, a total ionizing dose (TID) of $10 \mathrm{MGy}$ and $1 \mathrm{MeV}$ neutron equivalent fluence of $2 \times 10^{16} \mathrm{~cm}^{2}$ accumulated for the innermost silicon layer. Operation of the readout channel at low thresholds, around 1,000 electrons or lower, has to be envisaged, setting challenging requirements on noise and threshold dispersion performance. The chip will integrate the charge generated in the pixel sensor by crossing particles, amplify and digitize the signal, and send the hit information to the DAQ system after a LOA decision is received. It can operate at trigger frequencies up to $4 \mathrm{MHz}$ and has a latency of $12.5 \mu$ s and a power budget close to $0.5 \mathrm{~W} / \mathrm{cm}^{2}$.

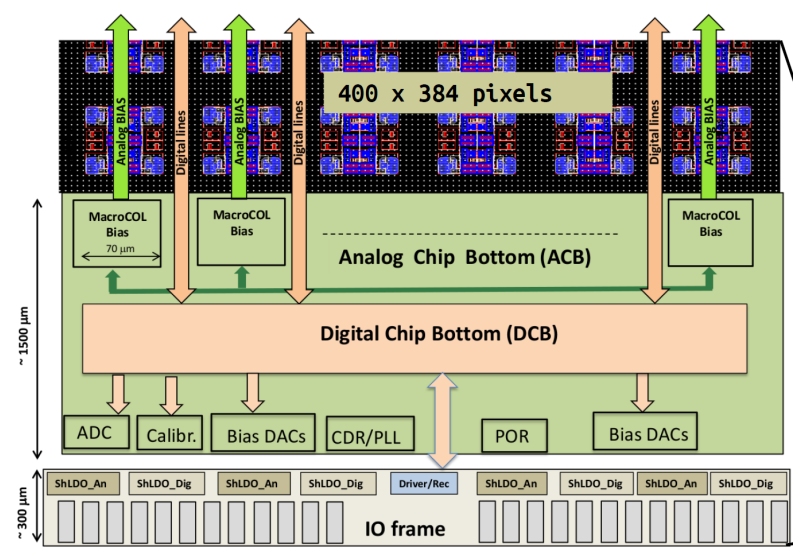

Fig. 2. Diagram of the pixel readout chip [4]. 
The upgraded ASIC includes advances in radiation tolerance, low power, and high rate capability. Each chip has an area of $20 \times 21 \mathrm{~mm}^{2}$ that handles 153,600 pixels and contains approximately $500 \mathrm{M}$ transistors. The pixel matrix is building up of $50 \times 48$ digital cores with 64 pixel channels each. The analog design must be fast enough to keep in-pixel pileup below the $1 \%$ hit loss specification, yet slow enough to allow time-over-threshold (ToT) amplitude digitization. The pixels within a core are further grouped into logical units for routing and logic area utilization. The analog front-ends are contained in units of $2 \times 2$ cores completely surrounded by synthesized logic. The digital core handles all processing of the frontend binary outputs, including masking, digital injection, charge digitization using ToT, storage of ToT values, latency timing, triggering, and readout. To keep the complexity of the cooling system for more than 5 billion channels at a minimum, the ASIC has been designed to operate in a serial power chain. Special power regulators called ShuLDOs are used to generate internal constant voltage rails while drawing a constant current from an external power source. Mitigation of single event upset will be implemented using triple redundant latches and/or continuous reconfiguration during operation.

\section{B. Calorimeter electronics}

A schematic diagram of the HL-LHC readout architecture for the Liquid Argon (LAr) calorimeters [11] is shown in Fig. 3. New readout Front-end Boards (FEB2), Calibration Boards, and LAr Signal Processor Boards (LASP) will be installed for the HL-LHC upgrade while the LAr trigger chain boards installed during the Phase-I upgrade will remain operational. FEB2s are designed to improve radiation tolerance and to increased the size of the trigger pipelines which will allow more advanced trigger algorithms to be applied. A triggerless readout scheme will be used and the detector signals will be amplified, shaped and split into two overlapping linear gain scales. Two shapers with low and high gains and two 14-bit analog-to-digital converters (ADCs) for each channel will be used to cover the 16-bit dynamic range. Both gain scales are digitized by an ADC, and this digital signal is multiplexed and sent off detector optically. The power consumption will remain to be $630 \mathrm{~mW}$ per channel.

A Front-End System-On-Chip (FESOC) approach is being pursued to reduce the system complexity. This ASIC will integrate 8 channels of pre-amplifiers and shapers, 16 channels of 14-bit ADCs, and a multiplexer/encoder/serializer unit. The stringent requirements on large dynamic range (from a few tens of $\mathrm{MeV}$ to a few $\mathrm{TeV}$ ), good linearity (at the per mille level for up to $10 \%$ of the dynamic range and at the level of a few percent at higher energies), high throughput (output data rate of $10 \mathrm{~Gb} / \mathrm{s}$ or $20 \mathrm{~Gb} / \mathrm{s}$ for $40-\mathrm{MHz}$ or $80-\mathrm{MHz}$ sampling rates, respectively), and radiation hardness make the design challenging. Each FEB2 will consist of 16 FESOCs and has 16 or 32 optical fibers operating at $10 \mathrm{~Gb} / \mathrm{s}$. The Calibration Boards inject accurate calibrated signals directly to the calorimeter cells. New LASPs will receive the full, digitized data stream from the FEB2s, apply digital filtering to the signals of each calorimeter cell, and buffer the data until a LOA signal is received.

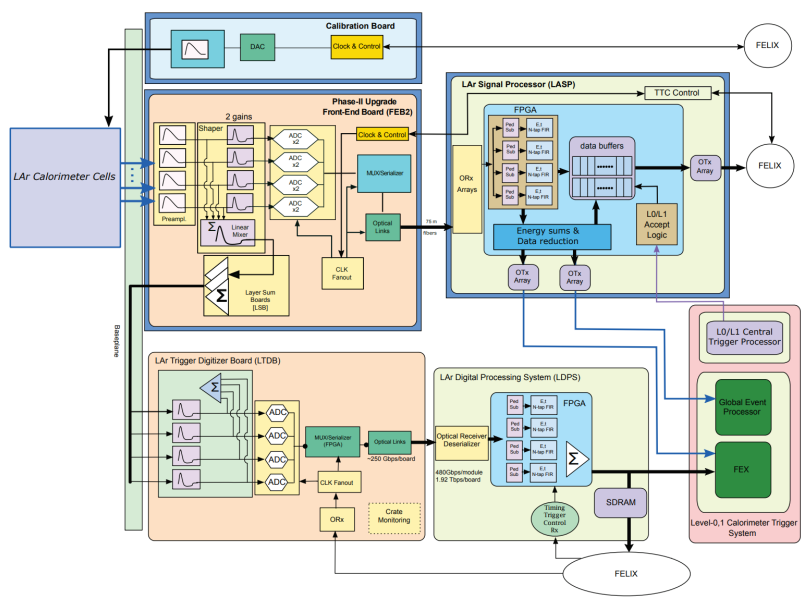

Fig. 3. Block diagram of the LAr calorimeter trigger and readout architecture [11].

\section{HGTD electronics}

The main purpose of the HGTD detector is to provide a $30-$ 50 ps time resolution per track, with a layout that guarantees on average 2 or 3 hits per track. In order to match the excellent performance of the sensor, the electronics' time jitter for an input charge of about $10 \mathrm{fC}$ is required to be smaller than 20 ps. The time walk should be smaller than 10 ps over the dynamic range after correction. To enable the possibility to set low thresholds for charges as low as $4 \mathrm{fC}$ due to the effects of irradiation, the cross-talk between channels should be kept below $5 \%$.

A custom ASIC (ALTIROC), which will be bump-bonded to the LGAD sensors, is being developed to meet the requirements on time resolution and radiation hardness. Each ASIC handles 225 readout channels. The most critical aspect concerning the time jitter is the design of the analog frontend electronics, which are composed of a voltage preamplifier followed by a fast discriminator. The measured timeof-arrival (ToA) and ToT are digitized using two time-todigital converters (TDCs), and stored in a local memory at the channel level. The TDC bin size is $20 \mathrm{ps}$ for the ToA measurement and $40 \mathrm{ps}$ for the ToT measurement. The Vernier delay line configuration is employed in the TDC for the ToA measurement. A graphic representation of the TDC working principle is shown in Fig. 4. This configuration consists of two lines, each composed of a series of controllable delay cells (140 ps for the slow line and 120 ps for the fast line). The number of cell stages necessary for the STOP signal (next rising edge of the $40 \mathrm{MHz}$ clock) to surpass the START signal (rising edge of the discriminator) represents the result of the time measurement with a step of $20 \mathrm{ps}$. The ToA and ToT information are transferred to the DAQ system only upon the LOA reception.

The HGTD will also be used as a luminosity measurement device by counting the number of hits within two different 
time windows. The two windows are centered at the expected arrival time of the particles from the collisions with their length adjustable via configuration parameters. The sideband for the longer time window will provide valuable information about the background. The number of hits registered in the sensor is then transmitted at a rate of $40 \mathrm{MHz}$ to allow unbiased, bunch-by-bunch measurements of the luminosity and the implementation of a minimum-bias trigger.

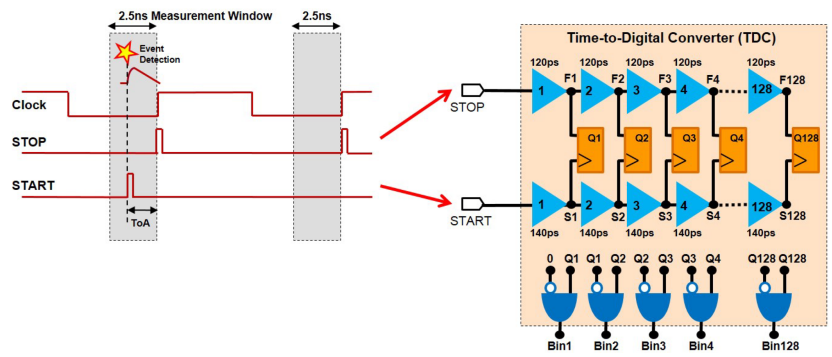

Fig. 4. Graphical representation of the working principle of the TDC for the HGTD detector [6].

\section{Muon electronics}

The precision coordinates measured by the MDT chambers will be used in the L0 trigger decision to improve the muon momentum measurements and to reduce the number of low momentum muons wrongly selected due to the moderate momentum resolution of the RPCs and TGCs. Figure 5 shows the block diagram of the firmware developed for the MDT trigger and readout processor board (LOMDT). MDT on-detector front-end electronics will be replaced and digitized hit data will be continuously transferred to the off-detector LOMDT board. The LOMDT needs to identify hits compatible with the region-of-interest and bunch-crossing timing provided by the muon trigger chambers. A fast track reconstruction algorithm will be performed on these matched hits for the refinement of the muon momentum measurement as the basis of the muon trigger decision. The LOMDT also buffers matched hits for 10 $\mu$ s and later transmits them to FELIX [12], upon the receiving of an L0A signal. In total 64 L0MDT boards are needed to match the 64 sectors for the MDT system. The available latency for the entire process of hit data reception and decoding, tube address mapping, drift-time to drift-distance conversion, hit extraction, pattern recognition, segment finding in each of the three stations (inner, middle, outer), and track fitting is $1.8 \mu \mathrm{s}$. Fast pattern recognition and track reconstruction algorithms running on FPGAs are critical. Two algorithms, Legendre Segment Finder and Compact Segment Finder, are currently under study and both options are found to stay within allocated latency budget.

To ensure continued operation of the present RPCs at the HL-LHC, these chambers will have to be operated at reduced performance, in order to respect the original design limits on currents and integrated charge. This can be achieved by reducing the gas gain through lowering the operating voltages. In the areas of highest backgrounds, the gas gain will have to be reduced to such low levels that the signal size is ten times smaller than that produced in the present RPC. More sensitive RPC front-end electronics are needed to detect pulses at the level of 1,000 electrons or lower. The new electronics needs to decrease the noise to obtain a low occupancy, decrease the rise time for improved time-of-flight capability, improve the performance of the discriminator and the time-to-digital converter, and minimize the power consumption to avoid the need for active cooling. An ASIC using the Silicon Bipolar Junction Transistors technology is currently under development.

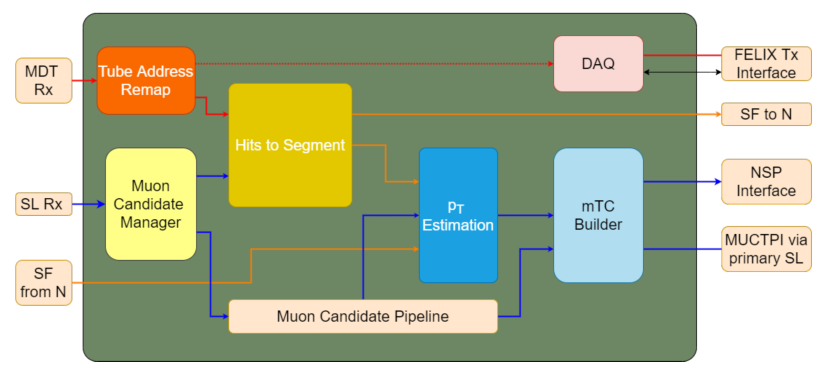

Fig. 5. Block diagram of the LOMDT firmware.

\section{CONCLUSiON}

The increases in both the integrated and the instantaneous luminosities also pose great challenges on electronics and the TDAQ system. The upgraded detector will have an unprecedented output data rate of up to $200 \mathrm{~TB} / \mathrm{s}$. Real-time processing of this large data volume in a short time period is extremely challenging. ATLAS is developing new sets of front-end and back-end electronics for all sub-detectors to meet these challenges. Detailed studies of these prototypes are ongoing and it is expected that all designs will be finalized in the next 2-3 years.

\section{REFERENCES}

[1] L. Evans, P. Bryant (Eds.), "LHC Machine", JINST, vol. 3, 2008, p. S08001.

[2] G. Apollinari et al., "High-Luminosity Large Hadron Collider Technical Design Report", CERN-2017-007-M, 2017.

[3] ATLAS Collaboration, "The ATLAS Experiment at the CERN Large Hadron Collider", 2008 JINST 3 S08003.

[4] ATLAS Collaboration, "Technical Design Report for the ATLAS Inner Tracker Pixel Detector", CERN-LHCC-2017-021, 2017.

[5] ATLAS Collaboration, "Technical Design Report for the ATLAS Inner Tracker Strip Detector", CERN-LHCC-2017-005, 2017.

[6] ATLAS Collaboration, "Technical Design Report: A High-Granularity Timing Detector for the ATLAS Phase-II Upgrade", CERN-LHCC2020-007, 2020.

[7] ATLAS Collaboration, "New Small Wheel Technical Design Report", CERN-LHCC-2013-006, 2013.

[8] ATLAS Collaboration, "Technical Design Report for the Phase-II Upgrade of the ATLAS Muon Spectrometer", CERN-LHCC-2017-017, 2017.

[9] ATLAS Collaboration, "Technical Design Report for the Phase-II Upgrade of the ATLAS TDAQ System", CERN-LHCC-2017-020, 2017.

[10] L. Gaioni, "Test results and prospects for RD53A, a large scale $65 \mathrm{~nm}$ CMOS chip for pixel readout at the HL-LHC", Nucl. Instr. Meth. A 936 (2019) 282.

[11] ATLAS Collaboration, "Technical Design Report for the Phase-II Upgrade of the ATLAS LAr Calorimeter", CERN-LHCC-2017-018, 2017.

[12] J. Anderson et al., JINST, 11 (2016) C01055. 